\title{
Lobectomía por VATS
}

\author{
PATRICIO RODRÍGUEZ D.*, FELIPE UNDURRAGA M.*,**, RAIMUNDO SANTOLAYA C.****, \\ DAVID LAZO P.* y JACQUELINE LÓPEZ R.*
}

\section{Video-thoracoscopic lobectomy}

Introduction: In the past two decades the advances in minimally invasive surgery have revolutionized surgical practices in all subspecialties. In pulmonary resections lobectomy by assisted videothoracoscopy (VATS) has demonstrated to have less perioperative complications, less inflammatory response and similar oncological results in comparison with lobectomy by thoracotomy. Methods and Patients: We present a retrospective study of 30 patients subjected to lobectomy by VATS. Diagnoses, complications intra and post-surgery, bleeding, conversion to thoracotomy, operatory time, lymphoadenectomy, pleurostomy and hospitalization time, were the variables analyzed. Results: $90 \%$ of the 30 patients $(n=27)$ had an oncological diagnosis, the remainder patients had benign lesions $(n=3)$, all of them presented bronchiectasis. TNM staging showed equal distribution for stages I and II. We obtained on average 13 nodes by lymphoadenoectomy (range: 9 to 18 nodes). Operatory time was 124 min on average. With respect to complications $50 \%$ of them $(n=3)$ bleed more than 1,000 $\mathrm{mL}$. The conversion rate in our series was $16.5 \%(n=5)$. Pleurostomy lasted 4.09 days on average (range: 2 to 9 days). There was no mortality in our series. Conclusion: In our series, lobectomy by VATS had equal range of intra and post- surgery complications as compared to toracotomy. We believe that our study demonstrates a benefit in the recovery time of our patients.

Key words: Lobectomy, videotoracoscopy, VATS.

\section{Resumen}

Introducción: En las últimas dos décadas los avances en cirugía mínimamente invasiva han revolucionado la práctica quirúrgica en todas las subespecialidades. En resecciones pulmonares la lobectomía por videotoracoscopía (VATS), en comparación con resecciones por toracotomía, ha demostrado, además del menor número de complicaciones perioperatorias, una menor respuesta inflamatoria y equivalencia en resultados oncológicos. Pacientes y Métodos: Se presenta una serie retrospectiva de 30 pacientes en quienes se realizó una lobectomía por VATS. Se analizan los resultados con respecto a complicaciones quirúrgicas y postquirúrgicas y considerando variables de diagnóstico, linfadenectomía, sangrado quirúrgico, conversión a toracotomía, tiempo operatorio, días de estadía y días con pleurostomía. Resultados: Las indicaciones quirúrgicas de nuestra serie fueron en un $90 \%(n=27)$ por patología neoplásica, el 10\% restante $(n=3)$ correspondió a patología benigna (bronquiectasias). La etapificación TNM dentro del subgrupo de cáncer pulmonar se distribuyó homogéneamente entre las etapas I y II. La linfadenectomía obtuvo un promedio de 13 ganglios con un rango entre 9 y 18. El tiempo quirúrgico promedio fue de 124 minutos. De las complicaciones, un $50 \%$ de estas $(n=3)$ se debieron a sangrado de más de $1.000 \mathrm{~mL}$. La tasa de conversión en nuestra serie es de 16,6\% (5 casos). El promedio de días con drenaje fue de 4,09 días con rango entre 2 y 9 días. No hubo mortalidad a 60 días. Conclusión: En la serie presentada se evidencia que la lobectomía por VATS es equivalente a la toracotomía con respecto al número de complicaciones. Creemos como equipo que presenta beneficios en el tiempo de recuperación del paciente.

Palabras clave: Videotoracoscopía, lobectomía, VATS.

* Servicio Médico-Quirúrgico, Instituto Nacional del Tórax.

** Departamento de Cirugía-Oriente, Facultad de Medicina, Universidad de Chile. 


\section{Introducción}

En las últimas dos décadas los avances en cirugía mínimamente invasiva han revolucionado la práctica quirúrgica en todas las subespecialidades. Por su complejidad técnica, en cirugía torácica el despegue de la técnica mínimamente invasiva ha sido más pausado.

La primera descripción de una toracoscopía fue hecha por Hans Christian Jacobeus en 1910, cuando se introdujo a la cavidad torácica por medio de un cistoscopio, cauterizando adherencias pleurales y facilitando el colapso pulmonar en el tratamiento de la tuberculosis ${ }^{1}$. Robert McKenna reportó la primera experiencia de una lobectomía por videotoracoscopía (VTC) en 1994². Desde entonces la lobectomía por VTC ha ganado popularidad llegando a realizarse, en el año 2006, hasta un 32\% de las lobectomías por cáncer pulmonar primario mediante este abordaje según la Sociedad de Cirujanos Torácicos de Estados Unidos $^{3}$. Se han descrito también otros procedimientos realizados por VTC como segmentectomías, neumonectomías, resecciones en manguito e incluso resecciones de pared torácica ${ }^{4}$.

La cirugía torácica video asistida (VATS), consiste en el acceso al tórax a través de una incisión de menos de $4 \mathrm{~cm}$ de longitud con o sin separación intercostal. Esto complementado con otros accesos mínimos para la visualización e instrumentalización endoscópica ${ }^{5}$. En este procedimiento -a diferencia de la toracotomía- no se seccionan los músculos.

Actualmente, en el cáncer pulmonar, la resección completa del tumor, vía toracotomía, es considerado el tratamiento quirúrgico de elección. Esta resección consiste en una segmentectomía, lobectomía o neumonectomía dependiendo de la ubicación y compromiso tumoral El enfrentamiento por medio de VATS y los resultados que se están publicando ha llevado a considerar este abordaje como una buena opción ${ }^{2-6}$.

El aun bajo número de lobecomías por VATS se explicaría por la curva de aprendizaje que esta técnica demanda y por las controversias oncológicas que existen al respecto ${ }^{6,7}$. En lo que hay relativo consenso en la literatura médica es en las lobectomías por cáncer pulmonar en etapas iniciales, donde la evidencia clínica señala que existe un menor número de complicaciones perioperatorias y menor repercusión biológica inflamatoria, además de equivalencia oncológica entre técnica por toracotomía versus VATS ${ }^{2-7}$.

Dentro de las ventajas de la lobectomía por VATS versus por toracotomía destaca el menor número de complicaciones perioperatorias, ade- más hay una menor respuesta inflamatoria (menor nivel de interleuquinas y de PCR) y menor reducción postoperatoria de CD4 y natural killers ${ }^{8-10}$. Esto podría ser el sustrato inmunológico que explique los mejores resultados perioperatorios que presenta VTC versus toracotomía. Para dilucidar si esta diferencia implica mayor sobrevida para los pacientes operados por VTC se requerirán estudios posteriores ${ }^{11}$.

Con respecto a la sobrevida en etapas iniciales de cáncer pulmonar, lobectomías por VATS han demostrado equivalencia en sobrevida a 3 y 5 años con respecto a resecciones por toracotomía ${ }^{12,13}$. Las linfadenectomías mediastinicas han demostrado ser equivalentes entre VTC y toracotomía con respecto al número de linfonodos resecados $^{13}$.

En el siguiente trabajo descriptivo, presentamos nuestra experiencia con 30 casos de lobectomías por VATS.

\section{Pacientes y Método}

Se realizó un análisis retrospectivo de 30 pacientes en quienes se efectuó una lobectomía por videotoracoscopía, entre enero de 2009 a diciembre de 2010 en pacientes operados en el Instituto Nacional del Tórax.

Las resecciones pulmonares por videotoracoscopía se realizan en múltiples patologías pulmonares como son cáncer pulmonar (primario y metástasis), tumores carcinoides, lesiones benignas, como tuberculosis, bronquiectasias, aspergilomas y lesiones congénitas. En la siguiente serie se realizaron resecciones pulmonares según los diagnósticos descritos en la Tabla 1. En los casos de cáncer pulmonar todos los pacientes estaban en etapa I o II a quienes se les realizó el estudio preoperatorio incluyendo, exámenes generales, estudio de función pulmonar, scanner pulmonar, bronco-fibroscopía y en algunos casos de sospecha de compromiso mediastínico se realizó PET/ CT (tomografía por emisión de positrones).

Tabla 1. Diagnósticos histológicos postquirúrgicos

\begin{tabular}{|lrc|}
\hline Diagnósticos & n & \% \\
Cancer pulmonar primario & & \\
- Adenocarcinoma & 15 & 50 \\
- Escamoso & 1 & 3,3 \\
- Células grandes & 4 & 13,3 \\
- Bronquioloalveolar & 1 & 3,3 \\
Carcinoide & 6 & 20 \\
Benignos & 3 & 10 \\
\hline
\end{tabular}


Los criterios de inclusión de pacientes de nuestro equipo son los detallados en la Tabla 2, pero en términos generales se planteó abordaje por VATS cuando las imágenes de las lesiones no comprometían grandes vasos y el estudio prequirúrgico (función pulmonar) anticipaba buena tolerancia a intubación monobronquial. En caso contrario cuando la TAC preoperatoria demostraba compromiso de grandes vasos, o antecedentes de cirugías previas se prefirió el abordaje por toracotomía.

Tabla 2. Lobectomía por VATS: criterios de inclusión

1 Lesión única

2 Sin compromiso de grandes vasos

$3 \mathrm{VEF}_{1}>1 \mathrm{~L}$

4 Sin quimioterapia, radioterapia o cirugía torácica previa

5 Estadio I y casos seleccionados de estadio II

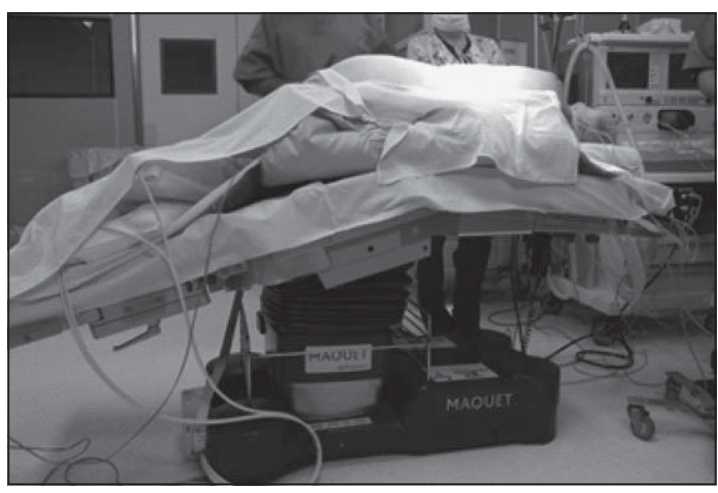

Figura 1. Imagen por dorsal de paciente en decúbito lateral derecho, para resección de tumor en lóbulo superior izquierdo.

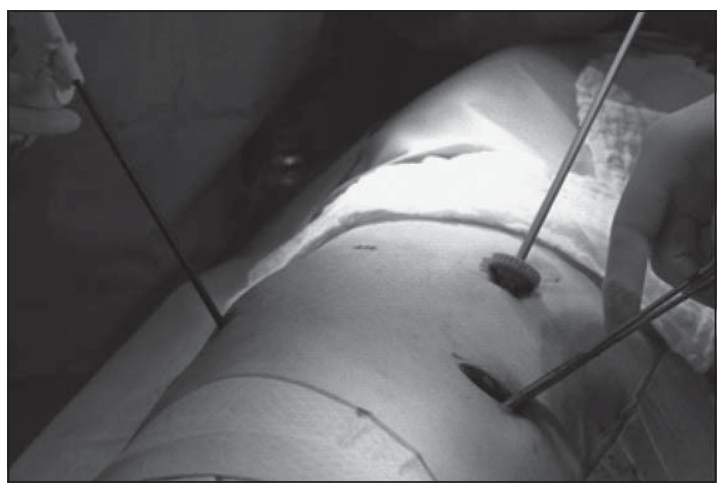

Figura 2a. Posicionamiento de trócares e insición accesoria. (i) trócar de $5 \mathrm{~mm}$ para óptica (ii), trócar de 5 $\mathrm{mm}$ de trabajo, (iii) insición accesoria de trabajo y para extracción de pieza.

\section{Posición de paciente e instrumental}

Bajo anestesia general e intubación selectiva monobronquial, se posiciona el paciente en decúbito lateral con el lado a operar hacia arriba. $\mathrm{Al}$ igual que para toracotomía posterolateral. Se realiza quiebre de la mesa quirúrgica a nivel de fosa lumbar (Figura 1). Los instrumentos para toracotomía están a disposición. Se ubican dos monitores a cada lado del paciente por cefálico. Cuando hay sólo un monitor, éste se coloca frente al cirujano, que se ubica por delante del paciente junto a la arsenalera y el ayudante se ubica por detrás del paciente y frente al cirujano.

\section{Posicionamiento de trócares}

Se accedió a la cavidad torácica mediante dos trócares y una incisión accesoria. Los trócares, ambos de $5 \mathrm{~mm}$, se posicionaron, dependiendo del tipo de lobectomía, en el séptimo y octavo espacio intercostal línea axilar posterior y media respectivamente. Por el trócar del octavo espacio en la línea axilar media se introduce una óptica de $30^{\circ}$. Eventualmente se utiliza un trócar accesorio de $5 \mathrm{~mm}$ colocado en el séptimo espacio intercostal en la línea escapular posterior, en caso de requerirse mayor tracción para la disección de hilio (Figuras 2a y 2b)

\section{VATS}

Una incisión accesoria -siempre menor a $4 \mathrm{~cm}$ - se realiza en el cuarto o quinto espacio intercostal en la línea axilar anterior. Por la cual se trabaja con disectores convencionales para resección de hilio. Esta incisión se utiliza también para extracción del lóbulo resecado (Figura 3).

\section{Lobectomía}

El posicionamiento de los trócares dependerá de la lobectomía a realizar. Si, en la primera

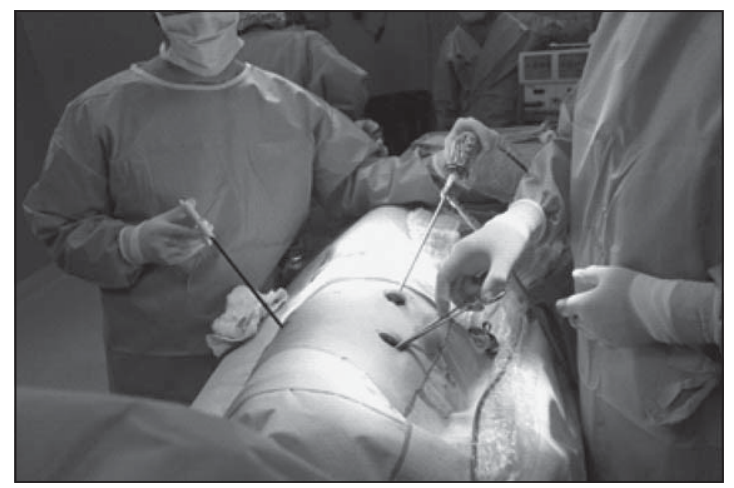

Figura 2b. Cirujano a la derecha, frente a la incisión accesoria y ayudante sosteniendo cámara y endoclinch para tracción. 


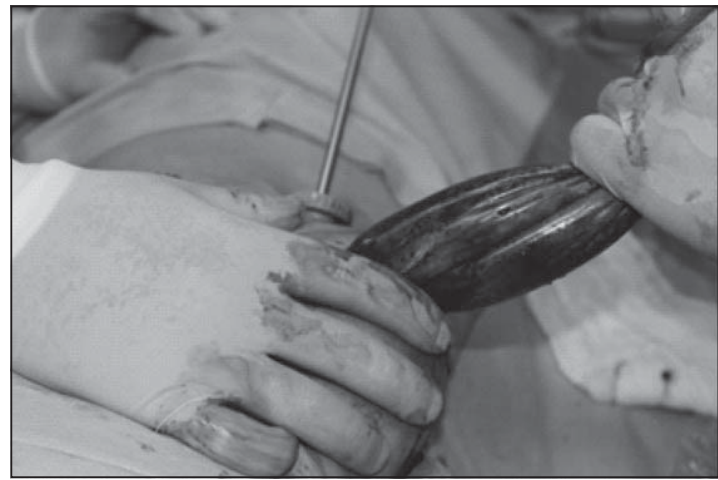

Figura 3. Extracción de la pieza operatoria por la incisión accesoria.

inspección de la cavidad torácica, se visualiza la presencia de adherencias pleuro-parietales éstas son resecadas por electrocoagulación o con ultrasonido en los casos en que se cuente con el equipo apropiado. La presencia de adherencias puede ser también un criterio de conversión a toracotomía, como sucedió con un paciente de nuestra serie. Se evalúan las características de la cisura y traccionando el parénquima pulmonar hacia posterior se procede a la disección hiliar anteroposterior usando el instrumental toracoscópico clásico, a través de la incisión accesoria. La disección ganglionar mediastínica se realizó mediante pinza Foerster endotorácica de las estaciones ganglionares $2 \mathrm{R}, 4 \mathrm{R}, 7,8,9$ y $10 \mathrm{R}$ para el lado derecho y 4L, 5, 6, 7, 8, 9 y 10 para el lado izquierdo. En todos los pacientes, con diagnóstico de cáncer pulmonar no células pequeñas, se realizó una disección linfática de regla. Los lóbulos resecados fueron extraídos a través de la incisión accesoria en bolsas impermeables.

\section{Vena, arteria y bronquio}

La rama de la vena pulmonar correspondiente a la lobectomía a realizar, se diseca mediante disección roma por disector toracoscópico o convencional, que se introduce por la incisión accesoria. Una vez liberada se secciona mediante endostapler con grapas de $3 \mathrm{~mm}$ para suturas vasculares.

El mismo procedimiento se realiza para disección de bronquio y arteria. En caso de bronquio se utilizó un endostapler de $4 \mathrm{~mm}$. Se describe que en algunos casos de disección venosa dificultosa se posiciona una ligadura para traccionar la vena y facilitar el paso del stapler (Figura 4).

\section{Cisura}

La cisura es seccionada posterior a la disección y resección de los vasos y el bronquio. Se realiza con un stapler a través de la incisión accesoria.

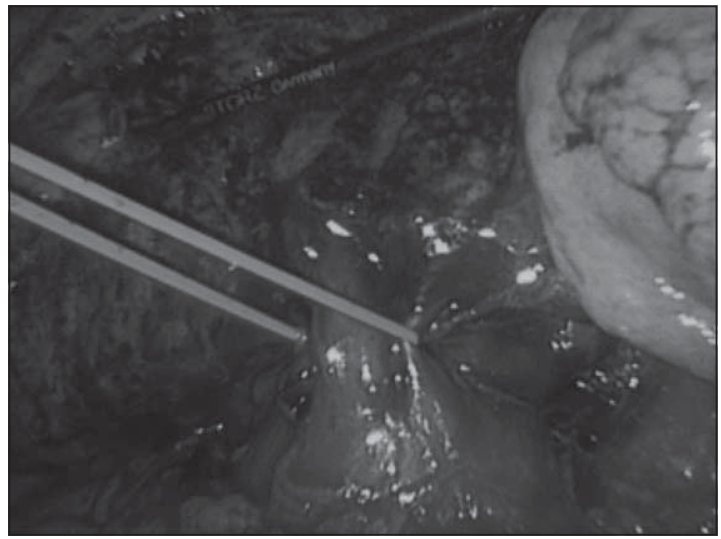

Figura 4. Tracción de vasos para lograr un mejor posicionamiento del stappler.

La pieza operatoria se extrae por esta misma incisión en bolsa impermeable (Figura 3).

\section{Resultados}

Del total de pacientes de la serie revisada, un $36,6 \%$ (11 pacientes) fueron hombres y 63,3\% (19 pacientes) fueron mujeres, con una media de 63 años (rango: 35 a 89 años). En el estudio prequirúrgico los pacientes de nuestra serie presentaron un $\mathrm{VEF}_{1}$ promedio de 2,46 L correspondiente a 98,5\% del valor teórico (rango 1,78 a 4,09L). Con respecto a la CVF el valor promedio fue de 3,05 L, 101\% del valor teórico (rango: 2,11 a 4,59 L).

Dentro de las indicaciones de lobectomía por videotoracoscopía, un $90 \%$ de nuestra serie fue por patología neoplásica dentro de ella 6 carcinoides (20\%). El resto correspondió a lesiones benignas $(n=3)$, todas ellas bronquiectasias (Tabla 1). En el subgrupo de cáncer pulmonar, la etapificación TNM se distribuyó homogéneamente entre las etapas I y II (Tabla 3). Con respecto a la ubicación anatómica de las lesiones (benignas y malignas) se distribuyeron con considerable diferencia en LSD ( $37 \%)$ como se muestra en la Tabla 4. La linfadenectomía, reglada según técnica descrita, obtuvo en promedio un total de 13 ganglios (rango: 9 a 18 ganglios). El tiempo quirúrgico fue de 124 minutos en promedio (rango: 60 a $280 \mathrm{~min})$.

Las complicaciones quirúrgicas las dividimos en quirúrgicas (13,3\%; $\mathrm{n}=4$; Tabla 5) y post-quirúrgicas (6,6\%; $n=2$; Tabla 6). Entre los pacientes que presentaron complicaciones quirúrgicas $(n=4)$, en todas hubo necesidad de conversión a toracotomía. De las complicaciones un $50 \%(n=3)$ se debió a sangrado de más de $1.000 \mathrm{~mL}$. La tasa de conversión en nuestra serie 
Tabla 3. Etapificación subgrupo cáncer*

\begin{tabular}{|lrc|}
\hline Etapa & n & \% \\
IA & 10 & 37 \\
IB & 9 & 33,3 \\
IIA & 7 & 26 \\
IIB & 1 & 3,7 \\
IIIA & 0 & 0 \\
IIIB & 0 & 0 \\
IV & 0 & 0 \\
\hline
\end{tabular}

*Corresponde a 27 pacientes.

Tabla 4. Lóbulos resecados

\begin{tabular}{|lcc|}
\hline Ubicación & $\mathbf{n}$ & $\mathbf{\%}$ \\
\hline LSD & 11 & 36,6 \\
\hline LID & 6 & 20 \\
LM & 2 & 6,6 \\
LSI & 6 & 20 \\
LII & 5 & 16,6 \\
\hline
\end{tabular}

$\mathrm{L}=$ lóbulo; $\mathrm{S}$ = superior; $\mathrm{M}=$ medio; $\mathrm{I}$ = inferior; $\mathrm{D}$ = derecho; $\mathrm{I}$ = izquierdo.

Tabla 5. Complicaciones intraoperatorias

\begin{tabular}{|lcc|}
\hline Complicación & $\mathbf{n}$ & $\mathbf{\%}$ \\
\hline Adherencia & 1 & 3,3 \\
Hemorragia mayor & 3 & 10 \\
Total & 4 & 13,3 \\
\hline
\end{tabular}

Tabla 6. Complicacionespostoperatorias

\begin{tabular}{|lll|}
\hline Complicación & n & \% \\
\hline Fístula bronquial & 1 & 3,3 \\
\hline Neumotórax & 1 & 3,3 \\
\hline Total & 2 & 6,6 \\
\hline
\end{tabular}

fue de 16,6\% (5 casos). El sangrado quirúrgico promedio fue de $650 \mathrm{~mL}$ (rango: 120 a $1.500 \mathrm{~mL}$ ). Se requirió transfusión sanguínea en dos casos, ambos dentro del grupo de conversión por hemorragia. Con respecto a los días con pleurostomía, su duración promedio fue de 4,09 días (rango: 2 a 9 días). En dos casos (6,6\%) se evidenciaron complicaciones postoperatorias. El primero por una fístula bronquial que requirió cirugía por toracotomía, con adecuada evolución, y un neumotórax de 30\%, que se manejó con pleurosto- mía. El promedio de tiempo de hospitalización de esta serie fue de 6,5 días (rango: 4 y 12 días). No hubo mortalidad a 60 días en la serie presentada.

\section{Discusión}

Las lobectomías por técnica abierta en estudios prospectivos randomizados han demostrado una tasa de complicaciones perioperatorias entre 32 y $37 \%$ con mortalidad entre 1 y $2 \%$. Los rangos menores se encuentran en centros de derivación de patología quirúrgica torácica ${ }^{14}$. Por su parte, en estudios retrospectivos las resecciones pulmonares mayores por videotoracoscopía, incluyendo las lobectomías, muestran una tasa de complicaciones entre el 10 y $20 \%$ con tasas de mortalidad de hasta el 2\% $\%^{15-17}$.

En resecciones por cáncer la evidencia indica que la lobectomía por videotoracoscopía en pacientes en etapas iniciales de cáncer no células pequeñas está asociada a un menor número de complicaciones postoperatorias ${ }^{16.17}$, a un menor impacto biológico en factores de inflamación ${ }^{8}$ y equivalencia en resultados oncológicos comparada con la lobectomía por técnica clásica mediante toracotomía ${ }^{7}$. Para alcanzar estos resultados equivalentes y los beneficios de la aproximación mínimamente invasiva se requiere alcanzar el plateau en las curvas de aprendizaje. Por ser la lobectomía por videotoracoscopía una técnica relativamente nueva, no existe consenso en el número que requiere la curva de aprendizaje requiriéndose según las publicaciones entre 20 y 50 casos para alcanzar el plateau de la curva de aprendizaje ${ }^{18}$.

Las curvas de aprendizaje para distintos procedimientos quirúrgicos han sido extensamente estudiadas. Se entiende por curva de aprendizaje superada, cuando el parámetro evaluado alcanza un valor estable y este es comparable con lo publicado ${ }^{19}$.

El análisis de múltiples parámetros, como los presentados en esta revisión: tiempo operatorio, sangrado, conversión a toracotomía, complicaciones postoperatorias, estadía hospitalaria, entre otros, son particularmente útiles en la evaluación de la técnica.

Nuestra revisión presenta la experiencia inicial en lobectomías por videotoracoscopía de nuestro equipo. En nuestro país desde el año 2009 se ha comenzado a utilizar esta técnica en forma relativamente frecuente. De los datos expuestos de la revisión de esta serie inicial se evidencia que tanto el porcentaje de complicaciones intraoperatorias $(13,3 \%)$ como de complicaciones postquirúrgicas $(6,6 \%)$ son similares a los publicados en diferentes series. También los días promedio con pleurostomía $(4,09)$, caen dentro del rango esperado ${ }^{20,21}$. En nuestra serie, destaca que el tiempo quirúrgico se encuentra en el rango inferior de lo publicado. El sangrado postquirúrgico se encuentra en el rango superior de lo publicado en distintas series, explicándose esto por un caso que requirió de conversión y reparación arterial. Frente a este caso se modificó la técnica quirúrgica. Comenzamos a realizar tracción de los vasos, tanto vena como arteria, con cintas vasculares, luego de disecados. 


\section{Conclusiones}

Como equipo, y en base a esta revisión, creemos que el enfrentamiento mediante videotoracoscopía debe ser considerado como primera opción en lesiones benignas que afecten un lóbulo o en cánceres T1-2, N0-1 y M0. El beneficio y acortamiento en tiempos de recuperación de pacientes se evidencia desde el postoperatorio inmediato.

Para llegar a conclusiones sólidas sobre esta técnica en nuestra realidad nacional se requiere la continuación de esta serie para lograr aumentar el número de pacientes y la realización de un estudio prospectivo nacional comparando los resultados de lobectomías por toracotomía versus videotoracoscopía, línea de trabajo actual de nuestro equipo.

\section{Bibliografía}

1.- JACOBAEUS H. U“” ber die Möglichkeit die Zystoskopie bei Untersuchung seröser Höhlungen anzuwenden. Münch. Med Wochenschr 1910; 57: 2.

2.- MCKENNA R J Jr. Lobectomy by video-assisted thoracic surgery with mediastinal node sampling for lung cancer. J Thorac Cardiovasc Surg 1994; 107: 879-82.

3.- BOFFA D J, ALLEN M S, GRAB J D, GAISSERT H, HARPOLE D, WRIGHT C. Data from the Society of Thoracic Surgeons General Thoracic surgery database: The surgical management of primary lung tumors. J Thorac Cardiovasc Surg 2008; 135: 247-54.

4.- MAHTABIFARD A, FULLER C B, MCKENNA R J Jr. Video-assisted thoracic surgery sleeve lobectomy: A case series. Ann Thorac Surg 2008; 85: S729-32.

5.- RODRÍGUEZ D, UNDURRAGA P. "Enfermedades Respiratorias”. Mediterráneo, Santiago, Chile, 2011.

6.- AMER K, KHAN A Z, VOHRA H A. Video-assisted thoracic surgery of major pulmonary resections for lung cancer: the Southampton experience, Eur J Cardiothorac Surg 2011; 39: 173-9.

7.- RUETH N M, ANDRADE R S. Is VATS Lobectomy Better: Perioperatively, Biologically and Oncologically? Ann Thorac Surg 2010; 89: S2107-11.

8.- CRAIG S R, LEAVER H A, YAP P L, PUGH G C, WALKER W S. Acute phase responses following minimal access and conventional thoracic surgery. Eur $\mathrm{J}$ Cardio- thorac Surg 2001; 20: 455-63.

9.- LEAVER H A, CRAIG S R, YAP P L, WALKER S. Lymphocyte responses following open and minimally invasive thoracic surgery. Eur J Clin Invest 2000; 30: 230-8.

10.- YIM A P, WAN S, LEE T W, ARIFI A. VATS lobectomy reduces cytokine responses compared with conventional surgery. Ann Thorac Surg 2000; 70: 243-7.

11.- RUETH N M, ANDRADE R S. Is VATS Lobectomy
Better: Perioperatively, Biologically and Oncologically? Ann Thorac Surg 2010; 89: S2107-11.

12.- YANG X, WANG S, QU J. Video-assisted thoracic surgery (VATS) compares favorably with thoracotomy for the treatment of lung cancer: A five-year outcome comparison. World J Surg 2009; 33: 1857-61.

13.- FARJAH F, WOOD D E, MULLIGAN M S, KRISHNADASAN B, HEAGERTY P, SYMONS R, et al. Safety and efficacy of video-assisted versus conventional lung resection for lung cancer. J Thorac Cardiovasc Surg 2009; 137: 1415-21.

14.- ALLEN M S, DARLING G E, PECHET T T, MITCHELL J, HERNDON J, LANDRENEAU R, et al. Morbidity and mortality of major pulmonary resections in patients with early-stage lung cancer: Initial results of the randomized, prospective ACOSOG Z0030 trial. Ann Thorac Surg 2006; 81: 1013-9; discussion: 101920.

15.- WHITSON B A, ANDRADE R S, BOETTCHER A, BARDALES R, KRATZKE R A, DAHLBERG P S, et al. Video-assisted thoracoscopic surgery is more favorable than thoracotomy for resection of clinical stage I non-small lung cancer. Ann Thorac Surg 2007; 83: 1965-70.

16.- FLORES R M, PARK B J, DYCOCO J, ARONOVA A, HIRTH Y, RIZK N, et al. Lobectomy by video-assisted thoracic surgery (VATS) versus thoracotomy for lung cancer. J Thorac Cardiovasc Surg 2009; 138: 11-8.

17.- HANDY J R Jr, ASAPH J W, DOUVILLE E C, OTT G Y, GRUNKEMEIER GL, WU Y. Does video-assisted thoracoscopic lobectomy for lung cancer provide improved functional outcomes compared with open lobectomy? Eur J Cardiothorac Surg 2010; 37: 451-5.

18.- PETERSEN R H, HANSEN H J. Learning thoracoscopic lobectomy. European Journal of Cardio-thoracic Surgery 2010; 37: 516-20.

19.- ZHAO H, BU L, YANG F, LI J, LI Y, WANG J. VideoAssisted Thoracoscopic Surgery Lobectomy for Lung Cancer: The Learning Curve. World J Surg 2010 34: 2368-72.

20-. AMER K, KHAN A, VOHRA H. Video-assisted thoracic surgery of major pulmonary resections for lung cancer: the Southampton experience. Eur J Cardiothorac Surg 2011; 39: 173-9. Epub. 2010.

21.-. KIM K, KIM H, PARK J, CHANG S, CHOI Y, KIM J, et al. Video-Assisted Thoracic Surgery Lobectomy: Single Institutional Experience With 704 Cases. Ann Thorac Surg 2010; 89: S2118-22.

\section{Correspondencia a:}

Dr. Patricio Rodríguez Duque

Email: prodriguez@clc.cl

Dr. Felipe Undurraga Machicao E-mail: felipeundu@gmail.com Instituto Nacional del Tórax José M. Infante 717. Santiago. Chile. 